\title{
Diffuse neutrino flux from blazars via cosmic-ray propagation in extragalactic medium
}

\author{
Saikat Das, ${ }^{a, *}$ Nayantara Gupta ${ }^{a}$ and Soebur Razzaque ${ }^{b}$ \\ ${ }^{a}$ Astronomy \& Astrophysics Group, Raman Research Institute, Bengaluru 560080, Karnataka, India \\ ${ }^{b}$ Centre for Astro-Particle Physics (CAPP) and Department of Physics, University of Johannesburg, P.O. \\ Box 524, Auckland Park 2006, South Africa \\ E-mail: saikatdas@rri.res.in
}

The origin of the cosmic neutrino signal measured by IceCube is still unknown. A general correlation between the sources of high-energy $\gamma$-ray sources with IceCube neutrinos is expected since hadronic interactions are the crucial precursors of both messengers at such high energies. Blazars dominate the extragalactic $\gamma$-ray sky. However, neutrinos originating from inside the blazar jets cannot account for more than $\sim 30 \%$ of the diffuse flux. We study ultrahigh-energy cosmic-ray contribution to the diffuse flux through photopion interactions on cosmic background photons. The extragalactic background light, consisting of IR/UV/optical photons require a proton energy threshold of $E_{\mathrm{th}}^{p, \pi} \approx 10^{17} \mathrm{eV}$ for pion production. The latter can yield $\mathrm{PeV}$ neutrinos. We analyze the total contribution from resolved and unresolved blazars by using a luminosity-dependent density evolution of blazars. The latest Fermi-LAT 4LAC catalog sheds light on the redshift distribution of the diffuse neutrino flux for resolved $\gamma$-ray blazars.

$37^{\text {th }}$ International Cosmic Ray Conference (ICRC 2021)

July 12th - 23rd, 2021

Online - Berlin, Germany

\footnotetext{
${ }^{*}$ Presenter
} 


\section{Introduction}

The IceCube neutrino observatory in Antarctica has established the existence of a diffuse flux of astrophysical neutrinos (from $\sim 10 \mathrm{TeV}$ to a few $\mathrm{PeV}$ ) [1]. The isotropic nature of the flux indicates the candidate sources to be of extragalactic origin. A single power-law can explain the observed all-flavor spectrum with a best-fit spectral index of $2.89_{-0.19}^{+0.2}[2,3]$. While $\gamma$-rays can be produced in both leptonic and hadronic processes, neutrinos are an exclusive probe of hadronic interactions. IceCube real-time alert program selects high-energy muon neutrino events $(\gtrsim 100$ $\mathrm{TeV}$ ) for rapid detection of electromagnetic counterparts arriving from the same direction [4]. The IceCube-170922A alert led to the $3 \sigma$ association of a flaring $\gamma$-ray blazar TXS $0506+056$ in spatial and temporal coincidence with a $\sim 0.3 \mathrm{PeV}$ muon track [5,6]. Some less significant candidate events with blazar-neutrino spatial coincidence have also been identified thereafter [7, 8]. Blazars are radio-loud AGNs with their relativistic jets pointed towards the observer. The emission region contains a tangled magnetic field and a relativistic plasma of electrons and cosmic rays. Electrons undergo radiative processes inside the jet, such as synchrotron, inverse-Compton (IC) scattering, etc. The seed photons for the IC process can be the synchrotron photons or external photons from the broad-line region, accretion disk, or dusty torus.

Cosmic rays accelerated in the jet are not cooled efficiently and can escape the source. However, they can also undergo pion production interactions inside the jet emission region with photons from synchrotron or IC spectrum, producing high-energy neutrinos [9-12]. An analysis by IceCube Collaboration with Fermi 2LAC catalog has found that such contribution can be maximum $\sim 30 \%$ of the diffuse flux [13]. In this work, we find out the neutrino flux from blazars due to interactions of cosmic rays above $10 \mathrm{PeV}$ with the cosmic background photons. Interaction with the UV/optical/IR photons will result in most neutrino events lying between a few $\mathrm{PeV}$ to tens of $\mathrm{PeV}$ [14]. The analysis with 8-yr Fermi-LAT 4LAC blazars gives us the fraction of diffuse neutrino flux as a function of redshift. We extend the study using a luminosity-dependent density evolution to find the cumulative flux from all resolved and unresolved blazars. An angular correlation of these neutrino events with the blazar population is difficult to obtain since cosmic rays are deflected from the source direction by the extragalactic magnetic field (EGMF).

\section{Results}

We denote the integrated $\gamma$-ray flux between $100 \mathrm{MeV}$ and $100 \mathrm{GeV}$ reported in the $4 \mathrm{LAC}$ catalog by the quantity $F_{100}$. The corresponding K-corrected $\gamma$-ray luminosity values are $L_{100}=$ $4 \pi d_{L}^{2} F_{100}(1+z)^{\Gamma-2}$, where $z$ is the redshift of the source, $d_{L}$ is the luminosity distance, and $\Gamma$ is the slope of the observed $\gamma$-ray spectrum. The $\gamma$-ray luminosity in the comoving frame (primed quantities) is Doppler boosted, $L_{100}=\left(\delta_{e}^{6} / \Gamma_{e}^{2}\right) L_{100}^{\prime}$ for FSRQs, and $L_{100}=\delta_{e}^{4} L_{100}^{\prime}$ for BL Lacs. Here $\delta_{e}$ and $\Gamma_{e}$ are the doppler factor and bulk Lorentz factor of the emitting plasma. The baryonic loading factor $\eta$ connects the cosmic-ray power with the $\gamma$-ray luminosity $L_{p}^{\prime}=\eta L_{100}^{\prime}$. We assume the observed $\gamma$-ray flux $F_{100}$ originates from only leptonic processes inside the source. We consider only protons with $E>10 \mathrm{PeV}$ are injected as cosmic rays. The cosmic-ray luminosity outside the jet (AGN frame) transforms as $L_{p}=\Gamma_{e}^{2} L_{p}^{\prime}$. Hence, in the observer frame, the scaling between $L_{p}$ and $L_{100}$ turns out to be $L_{p}=\Gamma_{e}^{2} L_{p}^{\prime}=\Gamma_{e}^{2} \eta L_{100}^{\prime}=\eta_{\mathrm{eff}} L_{100}$, where we assume $\delta_{e} \simeq \Gamma_{e}$, for jet opening 


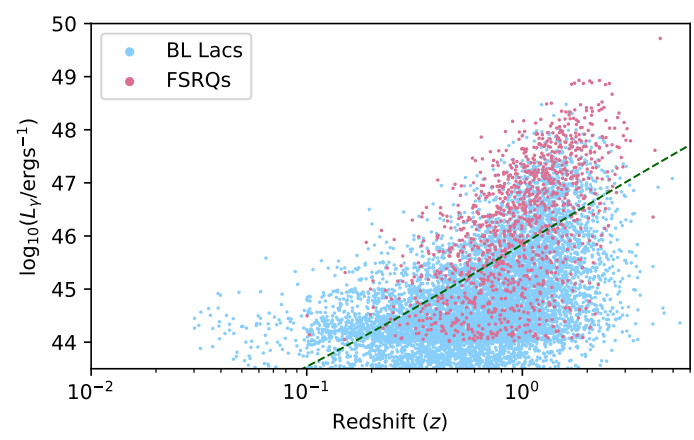

Figure 1: The distribution of blazars in luminosity-redshift space according to the luminosity function deduced in [20,21]. The dashed line separates the region into resolved and unresolved sources in Fermi-LAT survey. Figure adapted from Ref. [22]

angles $\theta_{j} \sim 1 / \Gamma_{e}$ and $\eta_{\mathrm{eff}}=\eta / \Gamma_{e}^{2}$ is the effective baryonic loading. The cosmic rays propagate and undergo $p \gamma$ interactions with EBL and CMB photons to produce $\mathrm{e}^{ \pm}, \gamma$-rays, and neutrinos. The secondary $\mathrm{e}^{ \pm}$and $\gamma$-rays initiate electromagnetic (EM) cascades down to $\mathrm{GeV}$ energies contributing to the IGRB flux measured by Fermi-LAT [15]. The neutrinos contribute to the isotropic diffuse neutrino background at $\mathrm{PeV}$ energies. Cosmic rays injected from the blazars are propagated using the CRPropa 3 simulation code to obtain the neutrino, $\gamma$-ray and cosmic ray flux at Earth [16]. We consider an injection spectrum of the shape $d N / d E \propto E^{-\alpha_{p}}$ and include all energy loss processes. The propagation of secondary EM particles, initiating the electromagnetic cascade, is solved using the DINT code [17, 18] and Gilmore et al. [19] EBL model.

Cosmic rays are injected from $10 \mathrm{PeV}$ up to a maximum energy $E_{p \text {,max }}$. The fraction of injected cosmic-ray energy $\left(\mathcal{E}_{p}\right)$, from a redshift $z$, carried away by cascade photons $\left(\mathcal{E}_{\gamma}\right)$ and secondary neutrinos $\left(\mathcal{E}_{v}\right)$ are given by $f_{v}(z)$ and $f_{\gamma}(z)$, respectively.

$$
\begin{aligned}
& f_{v}=\frac{\mathcal{E}_{v}(z)}{\mathcal{E}_{p}}=\frac{1}{\mathcal{E}_{p}} \times \int_{10 \mathrm{TeV}}^{E_{p, \max }} \epsilon_{\nu}\left(d N / d \epsilon_{\nu}\right) d \epsilon_{\nu} \\
& f_{\gamma}=\frac{\mathcal{E}_{\gamma}(z)}{\mathcal{E}_{p}}=\frac{1}{\mathcal{E}_{p}} \times \int_{10 \mathrm{MeV}}^{E_{p, \max }} \epsilon_{\gamma}\left(d N / d \epsilon_{\gamma}\right) d \epsilon_{\gamma}
\end{aligned}
$$

The value of $\mathcal{E}_{p}$ is fixed at all redshifts. These quantities signify the energy loss fraction of protons in various secondary channels. Cosmic magnetic fields can deflect the parent cosmic rays, smeared over a solid angle $\Omega$, thus resulting in a diffuse secondary flux. The normalization to the neutrino (and similarly, photon) luminosity is thus obtained as $L_{v}^{\mathrm{obs}}=f_{v} L_{p}=f_{v} \eta_{\mathrm{eff}} L_{100}$. Summing over all sources at all redshifts and different directions, the cumulative diffuse neutrino spectrum at Earth is

$$
F_{v}^{\mathrm{tot}}=\frac{1}{\Omega} \sum_{i}\left(\frac{L_{v}^{\mathrm{obs}}}{\Omega d_{L}^{2}}\right)_{i}\left[\mathrm{erg} \mathrm{cm}^{-2} \mathrm{~s}^{-1} \mathrm{sr}^{-1}\right]
$$

where $d_{L}$ is the luminosity distance corresponding to redshift $z$. The summation $i$ runs over the number of blazars in the sample space. We consider a conservative upper limit $\Omega=4 \pi$, which gives the minimum flux possible in this scenario. 

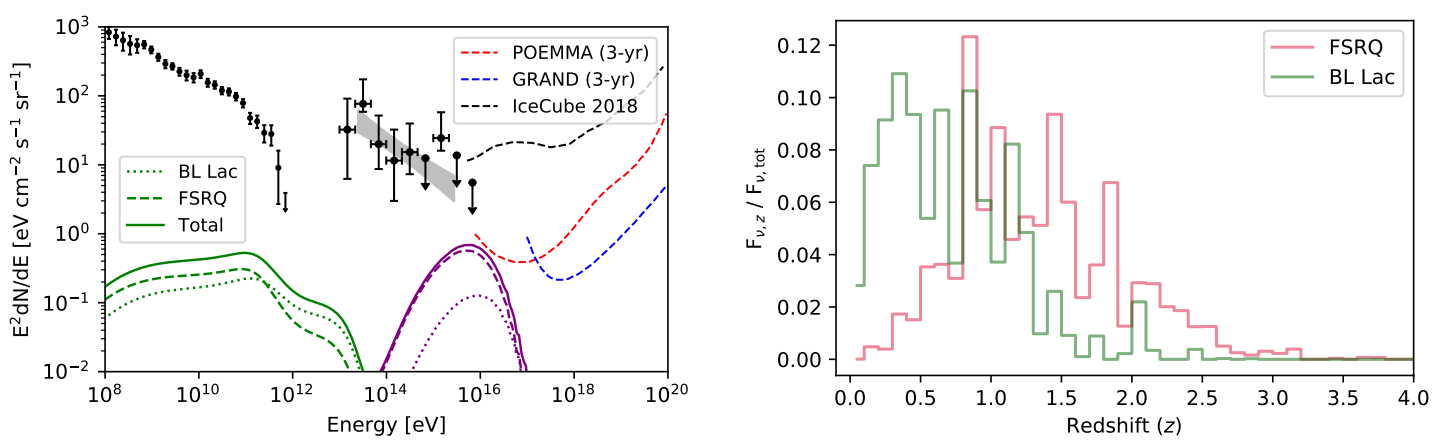

Figure 2: Left: The neutrino and IGRB flux from Fermi-detected blazars for $\eta_{\mathrm{eff}}=10.0$, and $E_{p \text {, } \max }=1$ EeV. Right: Fractional contribution to the neutrino flux from each redshift bin, relative to the individual flux from BL Lacs and FSRQs. Figure adapted from [22].

The luminosity function (LF) of blazars is modeled as a double power-law multiplied by the photon index evolution. We use the parametrization by $[20,21]$ to evaluate the distribution of BL Lac objects and FSRQs, including unresolved sources. At $z=0$, the number of sources $N$ per comoving volume $V_{c}$, emitted luminosity $L_{100}$ between $0.1-100 \mathrm{GeV}$, and slope of $\gamma$-ray flux $\Gamma$ is

$$
\Phi\left(L_{100}, z=0, \Gamma\right)=\frac{d N}{d L_{100} d V_{c} d \Gamma}=\frac{A}{\ln (10) L_{100}} \times\left[\left(\frac{L_{100}}{L_{*}}\right)^{\gamma_{1}}+\left(\frac{L_{100}}{L_{*}}\right)^{\gamma_{2}}\right]^{-1} g\left(\Gamma, L_{100}\right)
$$

The redshift evolution is incorporated by the factor $e\left(z, L_{100}\right)$, such that the luminosity dependent density evolution (LDDE) is represented as

$$
\Phi\left(L_{100}, z, \Gamma\right)=\Phi\left(L_{100}, z=0, \Gamma\right) \times e\left(z, L_{100}\right)
$$

The values of all parameters are obtained from the Refs. [20, 21]. We integrate Eqn. 5 over suitable ranges of luminosity, spectral index, and redshift. A total of 9172 blazars are obtained by integrating over the entire parameter range. The representative distribution of blazars in the $\ell-z$ space is shown in Fig. 1. The dashed line corresponds to a flux of $\phi_{\gamma}=1.25 \times 10^{-12} \mathrm{erg} \mathrm{cm}^{-2} \mathrm{~s}^{-1}$ and $\Gamma=2$, roughly separating the region into resolved and unresolved sources above and below, respectively. This threshold flux is chosen to match the 4LAC statistics of $\sim 2800$ observed blazars. For a detailed method of obtaining the distribution, please see Ref. [22].

The Fermi-LAT 4LAC catalog contains 655 FSRQs and 1067 BL Lacs. We first calculate the $\gamma$-ray and neutrino flux from these resolved sources. The sources inject protons between $10 \mathrm{PeV}-1$ $\mathrm{EeV}$, and $\alpha_{p}=2.6$. We consider the value of baryonic loading factor $\eta_{\mathrm{eff}}=10$. The analysis excludes 381 BL Lac objects lacking redshift information. The sources are binned in a two-dimensional $(\ell$, z) grid, where $\ell=\log _{10}\left(L_{100} / \mathrm{erg} \mathrm{s}^{-1}\right)$. We take the step sizes $\Delta \ell=0.5$ over the range 43.5 to 52.0 , and $\Delta z=0.1$ over the range 0.0 to 6.0 that covers the entire $4 \mathrm{LAC}$ catalog. The mean values $\ell_{m}$ and $z_{m}$ for a given bin are used to obtain the secondary neutrino and $\gamma$-ray flux according to Eqn. 3 . The number of sources $w$ in a grid provides the weight factor to the normalization of secondary fluxes. The resulting $\gamma$-ray and neutrino fluxes are shown in the left panel of Fig. 2. The neutrino spectrum peaks at an energy $E_{v} \approx 6 \mathrm{PeV}$. Our calculated flux is an order of magnitude lower than the IceCube upper limit at this energy. The right panel of Fig. 2 shows the fraction of neutrino luminosity 

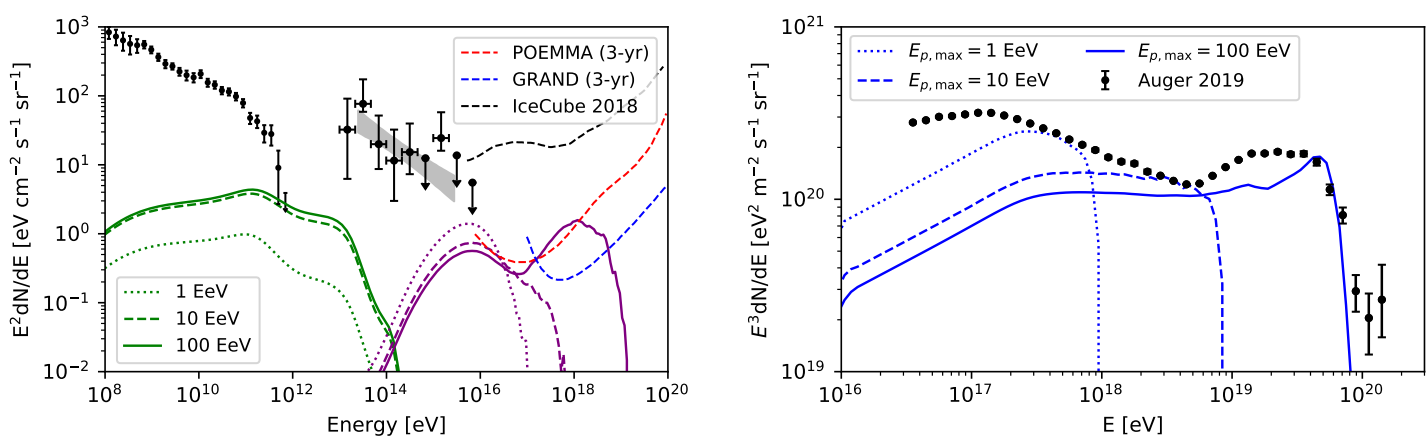

Figure 3: Left: The neutrino and IGRB flux, including the unresolved blazars, for the maximum values of $\eta_{\text {eff }}$ corresponding to $E_{p, \max }=1,10$, and $100 \mathrm{EeV}$. Right: The observed cosmic-ray spectrum at Earth for the maximum values of $\eta_{\text {eff }}$ corresponding to $E_{p \text {, max }}=1,10$, and $100 \mathrm{EeV}$, such that the UHECR flux is not violated. Figure adapted from Ref. [22].

coming from each redshift bin, individually for BL Lacs and FSRQs. The flux contribution from BL Lacs is approximately constant up to $z=1$ and then falls off sharply for higher $z$. Whereas the emission from FSRQs shows a peak near a redshift value of $z=1$.

The secondary fluxes obtained from the LDDE are shown in the left panel of Fig. 3. The number of blazars $w$ in each of the $(\ell, z)$ grid is calculated for the same values of $\Delta \ell$ and $\Delta z$ used in the preceding case. The dotted, dashed, and solid curves indicate the fluxes for the maximum allowed values of $\eta_{\mathrm{eff}}$ corresponding to each value of $E_{p, \max }$. The UHECR flux measured by the Pierre Auger Observatory (PAO) [23] puts an upper bound of $\eta_{\mathrm{eff}}=11.1,5.8$, and 4.4, for $E_{p \text {, } \max }=1,10$, and $100 \mathrm{EeV}$ respectively(cf. Fig. 3). We see that cosmic ray interactions can explain a little more than $10 \%$ of the IceCube flux upper limit at $\sim 6 \mathrm{PeV}$. An increase in the value of $E_{p \text {, max }}$ to $10 \mathrm{EeV}$ increases the cascade photon flux and saturates the IGRB background at $\mathrm{TeV}$ energies. To maintain the constraints put by IGRB measurements, the baryonic loading factor $\eta$ must be decreased for $E_{p, \max } \gtrsim 10^{19} \mathrm{eV}$. However, that in turn further decreases the neutrino flux at a few PeV energies. The neutrino flux obtained from an individual source, $F_{v} \propto \eta_{\mathrm{eff}} \propto \eta / \delta_{e}^{2}$, for a given value of $L_{100}$ and $E_{p, \max }$. We make the simplifying assumption $\delta_{e} \simeq \Gamma_{e}$ for the special case of $\theta \sim 1 / \Gamma_{e}$ for all blazars.

\section{Discussions and Conclusions}

We constrain the diffuse flux of PeV-EeV neutrinos originating from cosmic-ray interactions on EBL and CMB with $E_{p \text {,max }}$ up to 1-100 $\mathrm{EeV}$. Here, more luminous sources contribute more to neutrino and IGRB backgrounds. We assume that cosmic rays efficiently escape the system. We do not account for the sub-PeV neutrinos, which are expected to be dominated by neutrinos produced inside the high-energy sources. A strict $L_{p} / L_{100}$ correlation may not hold invariably for all sources. The "blazar sequence" predicts that BL Lacs with synchrotron and IC peak at higher energies are fainter in photon flux $[24,25]$. The low-luminosity counterparts can be more hadronically powered (higher $\eta_{\text {eff }}$ ) since the predicted SED peak energies are higher. The 4LAC catalog provides the redshift information for all the resolved FSRQs and most BL Lac objects. The deduced luminosity 
function of BL Lacs indicates that nearly $\sim 65 \%$ are yet to be identified. A majority of these are low-luminosity $\left(L_{\gamma}<10^{44} \mathrm{erg} \mathrm{s}^{-1}\right.$ ) counterparts. By including the unresolved sources, the neutrino flux increases by a factor of two at a few PeV. The values of $E_{p \text {,max }}$ considered in the study resembles the typical values obtained in the lepto-hadronic/hadronic modeling of blazar SEDs [26]. We see that beyond maximum acceleration energy of $\approx 10 \mathrm{EeV}$, the IGRB flux is saturated at TeV energies, thus requiring a lower baryon load, which reduces the neutrino flux at a few PeV. Depending on the normalization and the total number of blazars obtained in more updated luminosity-dependent density evolution functions [see, eg., 27], the estimates obtained here can change moderately.

\section{References}

[1] M. G. Aartsen et al. (IceCube Collaboration), Science 342 (2013), 1242856 [arXiv: 1311.5238].

[2] M. G. Aartsen et al. (IceCube Collaboration), Astrophys. J. 809 (2015) no.1, 98 [arXiv:1507.03991].

[3] A. Schneider [IceCube], PoS ICRC2019 (2020), 1004 [arXiv:1907.11266].

[4] M. G. Aartsen et al. (IceCube Collaboration), Astropart. Phys. 92 (2017), 30-41 [arXiv:1612.06028].

[5] M. G. Aartsen et al. (IceCube, Fermi-LAT, MAGIC, AGILE, ASAS-SN, HAWC, H.E.S.S., INTEGRAL, Kanata, Kiso, Kapteyn, Liverpool Telescope, Subaru, Swift NuSTAR, VERITAS and VLA/17B-403 Collaboration), Science 361 (2018) no.6398, eaat1378 [arXiv:1807.08816].

[6] M. G. Aartsen et al. (IceCube Collaboration), Science 361 (2018) no.6398, 147-151 [arXiv: 1807.08794].

[7] S. Garrappa et al. (Fermi-LAT, ASAS-SN and IceCube Collaboration), Astrophys. J. 880 (2019) no.2, 880:103 [arXiv:1901.10806].

[8] A. Franckowiak, S. Garrappa, V. Paliya, B. Shappee, R. Stein, N. L. Strotjohann, M. Kowalski, S. Buson, S. Kiehlmann and W. Max-Moerbeck, et al. Astrophys. J. 893 (2020) no.2, 162 [arXiv:2001.10232].

[9] K. Murase, Y. Inoue and C. D. Dermer, Phys. Rev. D 90 (2014) no.2, 023007 [arXiv:1403.4089].

[10] M. Petropoulou, S. Dimitrakoudis, P. Padovani, A. Mastichiadis and E. Resconi, Mon. Not. Roy. Astron. Soc. 448 (2015) no.3, 2412-2429 [arXiv:1501.07115].

[11] A. Palladino, X. Rodrigues, S. Gao and W. Winter, Astrophys. J. 871 (2019) no.1, 41 [arXiv:1806.04769].

[12] X. Rodrigues, J. Heinze, A. Palladino, A. van Vliet and W. Winter, Phys. Rev. Lett. 126 (2021) no.19, 191101 [arXiv:2003.08392]. 
[13] M. G. Aartsen et al. (IceCube Collaboration), Astrophys. J. 835 (2017) no.1, 45 [arXiv:1611.03874].

[14] O. E. Kalashev, A. Kusenko and W. Essey, Phys. Rev. Lett. 111 (2013) no.4, 041103 [arXiv:1303.0300].

[15] M. Ackermann et al. (Fermi-LAT Collaboration), Astrophys. J. 799 (2015), 86 [arXiv:1410.3696].

[16] R. Alves Batista, A. Dundovic, M. Erdmann, K. H. Kampert, D. Kuempel, G. Müller, G. Sigl, A. van Vliet, D. Walz and T. Winchen, JCAP 05 (2016), 038 [arXiv: 1603.07142].

[17] S. Lee, Phys. Rev. D 58 (1998), 043004 [arXiv:astro-ph/9604098].

[18] C. Heiter, D. Kuempel, D. Walz and M. Erdmann, Astropart. Phys. 102 (2018), 39-50 [arXiv:1710.11406].

[19] R. C. Gilmore, R. S. Somerville, J. R. Primack and A. Dominguez, Mon. Not. Roy. Astron. Soc. 422 (2012), 3189 [arXiv:1104.0671].

[20] M. Ajello, M. S. Shaw, R. W. Romani, C. D. Dermer, L. Costamante, O. G. King, W. MaxMoerbeck, A. Readhead, A. Reimer and J. L. Richards, et al. Astrophys. J. 751 (2012), 108 [arXiv:1110.3787].

[21] M. Ajello, R. W. Romani, D. Gasparrini, M. S. Shaw, J. Bolmer, G. Cotter, J. Finke, J. Greiner, S. E. Healey and O. King, et al. Astrophys. J. 780 (2014), 73 [arXiv:1310.0006].

[22] S. Das, N. Gupta and S. Razzaque, Astrophys. J. 910 (2021) no.2, 100 [arXiv:2012.13877].

[23] A. Aab et al. (Pierre Auger Collaboration), [arXiv:1909.09073].

[24] G. Fossati, L. Maraschi, A. Celotti, A. Comastri and G. Ghisellini, Mon. Not. Roy. Astron. Soc. 299 (1998), 433-448 [arXiv:astro-ph/9804103].

[25] G. Ghisellini and F. Tavecchio, Mon. Not. Roy. Astron. Soc. 387 (2008), 1669 [arXiv:0802.1918].

[26] M. Boettcher, A. Reimer, K. Sweeney and A. Prakash, Astrophys. J. 768 (2013), 54 [arXiv:1304.0605].

[27] Y. Qu, H. Zeng and D. Yan, Mon. Not. Roy. Astron. Soc. 490 (2019) no.1, 758-765 [arXiv:1909.07542]. 